\title{
Anomalous nature of gravity at quantum realm
}

\author{
Kapil Chandra ${ }^{1}$ \\ Department of physics, University of Bastar, Dharampura 1, Jagdalpur [CG], India, 494226
}

\begin{abstract}
Gravity is a long range force, however, we studied its behavior at the scale where quantum effect comes in act by applying quantum - gravitational effect on this force. We reported, under this effect the classical gravity doesn't quantized instead its inverted and contrary to classical gravity it doesn't bind matter together and behaves anomalously.
\end{abstract}

Keywords: quantum — gravitational effect, gravity and inverted gravity, vacuum energy density.

Newton's classical gravity is a fundamental force of nature and dominant at large scale. This is responsible for binding together the matthers, galaxies and planets. Attractiveness of this force has been successfully tested at scale of millimetre range but beyond this range i. e. at shorter scale length, more precisely to say at quantum realm we don't have any idea about its nature; whether its attractive or repulsive?

Besides, it has been a challenge to determine and describe how it reconcile the quantum effect; either being quantized or remains a classical force? These open questions needs to be fixed to understand the behaviour of gravity in the perspective of quantum physics. The answer of these questions are so important in the sense that it might sheds light on the origin of this force.

It is believed that quantum theory of gravity might fix these open question, however, there is intensive and desperate search for this theory [1] but its still in progress and we don't have complete description of this theory till date $[\underline{2}, \underline{3}]$. It gives us opportunity to postulate some alternative hypothesis to meet these open questions.

In this paper, a different approach has been initiated instead of pursuing quantum theory of gravity, we simply tried to find an interrelation between gravitational and Planck constant which is done by applying quantum - gravitational effect on classical gravity. We expected this interrelation will be equivalent to a quantum form of gravity someway and obtained result will explain the behavior gravity under influence of quantum effect at quantum realm.

On order to derive that desired relation, initially we presumed that there is a numerical and dimensional balance between classical and quantum

\footnotetext{
1 e-mail: kapil.chandra@gov.in
}

force and empirically propose a force balance equation as written below,

$$
G \frac{m^{2}}{r^{2}} \times \frac{c^{4}}{G}=\frac{h c}{r^{2}} \times \frac{m^{2} c^{3}}{h}
$$

here, all used constants and variables holds its usual meanings.

In this expression all terms has same dimension as force and denotes different form of force and shows a balance between quantum and classical force, thus we named it "Force Balance Equation" (hereafter we abbreviated it as FBE).

First two expression of this FBE denotes classical force since it possess only universal gravitational constant and these are well known so doesn't need descriptions more.

Rest two mathematical expression has Planck's constant and denotes quantum of force. Its origin is due to quantization of space/mass obviously, however, these equations can estimate the theoretical numerical value of strong nuclear force [4] by replacing $\boldsymbol{R}$ with size of nucleus and $\boldsymbol{m}$ with the mass of proton or meson respectively.

It will be worthy to describe that this proposed FBE is in consistent with other existing theories; it can be seen if we theoretically derive an expression for relative strength of gravity to quantum of force or strong force, it gives us followings,

$$
\frac{h c}{R_{Q}^{2}}=\left(\frac{h c}{G m_{Q}^{2}}\right) G \frac{m^{2}}{R^{2}}
$$

the bracket terms is a constant quantity. It's value is nearly 39 orders of magnitude if we take $\boldsymbol{m} \boldsymbol{Q}$ as mass of proton and suggest strong force or nuclear force is 
stronger to gravity by 39 orders of magnitude in quantity and this quantity is gravitational coupling constant which is well accepted in theoretical physics.

Now, it is notable that mass and space are only used variable in FBE, however, relation between these variables are followings,

$$
R=\frac{G m}{c^{2}}
$$

it possess universal gravitational constant thus this relation is classical by nature. Another possible relation is,

$$
R=\frac{h}{m c}
$$

since, it possess Planck constant thus its quantum relation between used variables.

Next, one can observe from FBE that the quantum force possess only Planck constant whereas classical force has only gravitational constant therefore on order to derive an expression of force which possess both constant i.e. a quantum - gravitational form of force, here we presumed that the mass/space associated with quantum mechanics is same, equal, replaceable and interchangeable with the mass/space associated with classical mechanics.

However, initially we assumed that all the variables of FBE a quantum entity and substituted the quantum relation between mass and space in it i.e. Eq. (4) in Eq. (1); it doesn't give any new expression, its unchanged.

But, when we assumed all the variables as classical entity and substituted the classical relation between the mass and space in FBE i.e. Eq. (3) in Eq. (1), it gives,

$$
\frac{r^{2} c^{8}}{m^{2} G^{3}} \times \frac{c^{4}}{G}=\frac{h c^{5}}{G^{2} m^{2}} \times \frac{r^{2} c^{7}}{h G^{2}}
$$

it is the desired expression of force and these all are new expression of forces in fundamental physics. This expression is just inverted form of our proposed FBE thus we named it "inverted FBE" anyway.

One can observe that the RHS of this equation consists from two mathematical expression of force which has both Planck constant and gravitational constant thus its a quantum - gravitational terms, this is only the reason that this substitution is quantum gravitational effect itself.

This substitution and inversion of FBE is valid because if we take a relation $E=F . R$ where $R$ is space, and energy $E=k_{B} T$ where $k_{B}$ is Boltzmann constant, The first term of RHS of inverted FBE give the mathematical expression of Hawking temperature [5] and subsequent terms denotes the mathematical expression for vacuum energy density or dark energy density of vacuum in Planck unit what was earlier empirically proposed by Zeldovich himself [6]. These both entities are well accepted and has connection with other established theories which are re-derived so that the empirically proposed FBE and its inversion is in consistent with existing other theories indeed.

A remarkable aspect of this quantum gravitational effect is that the quantum of force gives a quantum - gravitational form of force but classical gravity didn't gives a quantum form of gravity instead its inverted, as written below,

$$
F=\frac{R^{2} c^{8}}{m^{2} G^{3}}
$$

it's an inverted form of Newton's classical gravity and a new form of force in physics. Contrary to classical gravity, its numerical value is proportional to the square of distance and inversely proportional to square of mass, so when distance is large the amount of force is large, however, it is not a dominant force anymore at quantum scale since at this scale distance is short definitely. It might be strong at large scale only.

The role played by this force in physics is yet to be studied but it has been supposed that this force might be responsible for cosmic acceleration [7] because this phenomenon is predicted at large scale length so this force might be a dominant force over that scale.

If this inverted gravity has some role in cosmic acceleration so one can predict that the lighter bodies accelerates away with faster speed, this needs to be verified by any physical observations in future.

In summary, classical gravity exhibits an anomalous behavior at quantum scale by inverting itself and not binding the matter together. This is very surprising nature of gravity.

The mathematical expression of this new form of force "the inverted gravity" suggests, when the distance is larger the force is greater, however, it might be potential candidate to explain the accelerated expansion of universe, further research recommended in this area to fix its viable role in cosmic acceleration.

Acknowledgments: I would like to say thanks to Dr. SK Verma for his helpful discussions and suggestions.

\section{References}

[1] C. Kiefer, in Approaches to Fundamental Physics, Lecture Notes in Physics edited by I.O. Stamatescu., E. Seiler (Springer, Heidelberg, 2007)

[2] L. J. Garay, arXiv:gr-qc/9403008.

[3] N. Pinto-Neto, W. Struyve, arXiv: 1801.03353v1[gr-qc] and referenced therein.

[4] H. Yukawa, J. Phys.-Math. Soc. Jpn.7,195 (1933).

[5] S. W. Hawking, Rev. Mod. Phys. 61, 1 (1972)

[6] Y. B. Zeldovich, JETP Lett. 6, 316 (1967).

[7] A. Riess at el., Astron. J 116, 1009 (1999); S. Perlmutter at el., Ap. J. 517, 565 (1999). 